\title{
Intersection theory in complex analytic geometry
}

\author{
by Piotr Tworzewski (Kraków)
}

\begin{abstract}
We present a construction of an intersection product of arbitrary complex analytic cycles based on a pointwise defined intersection multiplicity.
\end{abstract}

1. Introduction. Let $X$ and $Y$ be irreducible analytic subsets of a complex manifold $M$ of dimension $m$ (in this paper all manifolds are assumed to be second-countable). We say that the intersection of $X$ and $Y$ is proper if $\operatorname{dim}(X \cap Y)=\operatorname{dim} X+\operatorname{dim} Y-m$. Then we have the intersection product $X \cdot Y$ of $X$ and $Y$ in $M$, denoted also by $X \cdot_{M} Y$, which is an analytic cycle on $M$ defined by the formula

$$
X \cdot Y=\sum_{C} i(X \cdot Y ; C) C,
$$

where the summation extends over all analytic components $C$ of $X \cap Y$ and $i(X \cdot Y ; C)$ denotes the intersection multiplicity along the component $C$ in the sense of R. Draper ([Dr], Def. 4.5, see also [W1]).

This paper contains a proposal of the extension of this well-known definition to the case of improper (excess) intersections. In the presented theory the intersection product $X \bullet Y$ is an analytic cycle

$$
X \bullet Y=\sum_{j \in J} \alpha_{j} C_{j},
$$

i.e. $\alpha_{j} \in \mathbb{Z}$ for $j \in J$ and $\left\{C_{j}\right\}_{j \in J}$ is a locally finite family of irreducible analytic subsets of the manifold $M$.

The case of an improper isolated point of intersection has been worked out in [ATW]. Intersection multiplicities for irreducible (proper or improper) components are algebraically investigated in [AM1], [AM2], and [AM3]. However, a full construction of an intersection product was not finished.

1991 Mathematics Subject Classification: 14C17, 32B10, 32C25, 32C99.

Key words and phrases: analytic cycles, multiplicity of proper and improper intersection.

This research was partially supported by DFG and by KBN Grant 2 P03A 06108. 
In this paper we assign to every point $a \in M$ a geometrically defined intersection multiplicity $d(a)$ of $X$ and $Y$ at the point $a$, by analogy with the Stückrad-Vogel cycle (cf. [G1], [G2], [SV], [V]). The function $d: M \ni a \rightarrow$ $d(a) \in \mathbb{Z}$ is analytically constructible (Theorem 6.2) and defines a required intersection product $X \bullet Y$ (Definition 6.3). In the next natural steps we can extend this definition to the case of two (by $\mathbb{Z}$-bilinearity) or more analytic cycles.

The organization of this paper is as follows. Sections 2 and 3 are of preparatory nature; we collect together some facts on analytic cycles and derive their consequences for use in other parts. In Sections 4 and 5 our main results are proved, and then used in Section 6 to the main construction of a general intersection product.

This paper is to be treated as the first in a series on this subject. It is meant to lay down the necessary foundations. As for prerequisites, the reader is expected to be familiar with the theory of proper intersections of analytic sets in complex analytic geometry. Draper's paper [Dr] is our best reference. We shall use the notation and basic results of [Dr] (see also [Ch], Chapter 2).

2. Cycles, multiplicities, and constructible functions. In this paper analytic means complex analytic, and manifold means a complex manifold satisfying the second axiom of countability. A function $f: X \rightarrow Y$ from a topological space $X$ to an ordered set $Y$ is called upper semicontinuous if for every $y \in Y$ the set $\{x \in X: f(x) \geq y\}$ is closed in $X$. Usually we consider $Y=\mathbb{N}^{p}, p \geq 1$, with the lexicographic ordering $\left(^{1}\right)$.

An analytic cycle on a manifold $N$ is the formal sum

$$
A=\sum_{j \in J} \alpha_{j} C_{j}
$$

where $\alpha_{j} \neq 0$ for $j \in J$ are integers and $\left\{C_{j}\right\}_{j \in J}$ is a locally finite family of pairwise distinct irreducible analytic subsets of the manifold $N$.

The analytic set $\bigcup_{j \in J} C_{j}$ is called the support of the cycle $A$ and is denoted by $|A|$. The sets $C_{j}$ are called the components of $A$ with multiplicities $\alpha_{j}, j \in J$. We say that the analytic cycle $A$ is positive if $\alpha_{j}>0$ for all $j \in J$. If all the components of $A$ have the same dimension $k$, then $A$ will be called a $k$-cycle.

We denote by $\mathcal{G}(N)$ the set of all analytic cycles on $N$ with the natural structure of a commutative group. For $k \in \mathbb{N}$ the subgroup of $k$-cycles of $\mathcal{G}(N)$ will be denoted by $\mathcal{G}^{k}(N)$. Observe that the set of indices $J$ has to be at most countable and that 0 (neutral element) corresponds to $J=\emptyset$.

$\left({ }^{1}\right)$ Notice that if $f=\left(f_{1}, \ldots, f_{p}\right)$, then there are no simple relations between such a semicontinuity of $f$ and the classical semicontinuity of its real components $f_{1}, \ldots, f_{p}$. 
Now, let $N$ be an $n$-dimensional manifold and let $Z$ be a pure $k$-dimensional analytic subset of $N$. For $c \in N$ we denote by $\nu(Z, c)$ the degree of $Z$ at the point $c$ (cf. [Dr], p. 194). This degree is equal to the classical algebraic Samuel multiplicity, and to the so-called Lelong number of $Z$ at the point $c$. In this paper we will consider a natural extension of this definition to the case of an arbitrary analytic cycle. Namely, if $A=\sum_{j \in J} \alpha_{j} C_{j}$ is an analytic cycle on $N$, then the sum

$$
\nu(A, c)=\sum_{j \in J} \alpha_{j} \nu\left(C_{j}, c\right)
$$

is well defined, and we call it the degree of the cycle $A$ at the point $c$.

For an analytic cycle $A$ there exists a unique decomposition

$$
A=T_{(n)}+T_{(n-1)}+\ldots+T_{(0)},
$$

where $T_{(j)}$ is a $j$-cycle for $j=0, \ldots, n$. We define the extended degree of $A$ at $c$ by the formula

$$
\widetilde{\nu}(A, c)=\left(\nu\left(T_{(n)}, c\right), \ldots, \nu\left(T_{(0)}, c\right)\right) \in \mathbb{Z}^{n+1} .
$$

Denote by $\nu(A)$ and $\widetilde{\nu}(A)$ the functions

$$
\nu(A): N \ni x \rightarrow \nu(A, x) \in \mathbb{Z}, \quad \widetilde{\nu}(A): N \ni x \rightarrow \widetilde{\nu}(A, x) \in \mathbb{Z}^{n+1} .
$$

Observe that $\nu(A, x)=\widetilde{\nu}(\widehat{A}, x)$, where $\widehat{\mu}$ denotes the sum of the coordinates of $\mu \in \mathbb{Z}^{n+1}$.

If $A$ is positive, then by ([Wh], p. 237) (see also [Ch], p. 127), the function $\nu(A): N \rightarrow \mathbb{N}$ is upper semicontinuous in the Zariski topology on $N$. By a standard calculation one can show that also $\widetilde{\nu}(A): N \rightarrow \mathbb{N}^{n+1}$ is upper semicontinuous if in $\mathbb{N}^{n+1}$ we consider the lexicographic ordering.

Finally, let us recall that a function $f: N \rightarrow \mathbb{C}$ is called analytically constructible if its graph is an analytically constructible subset of $N \times \mathbb{C}$ (see [Ł2], IV, §8). Define

$$
\mathcal{K}(N)=\{f: N \rightarrow \mathbb{C}: f(N) \subset \mathbb{Z}, f \text { is analytically constructible }\} .
$$

Observe that $f: N \rightarrow \mathbb{Z}$ belongs to the class $\mathcal{K}(N)$ if and only if all the fibres of $f$ are analytically constructible subsets of $N$.

We end this section with the following useful

(2.1) Proposition. Let $N$ be a manifold. Then:

(1) if $A \in \mathcal{G}(N)$, then $\nu(A)$ is an analytically constructible and locally bounded function,

(2) if $f \in \mathcal{K}(N)$ and if $C$ is an irreducible analytic subset of $N$, then there exists $\mu \in \mathbb{Z}$ such that $\overline{C \cap f^{-1}(\mu)}=C$,

(3) the function $\nu: \mathcal{G}(N) \ni A \rightarrow \nu(A) \in \mathcal{K}(N)$ is an additive bijection. 
Proof. If $C$ is an irreducible analytic subset of $N$, then $\nu(C)$ is an upper semicontinuous function in the Zariski topology of $N$. This implies (1) in the case $A=C$. The general case follows easily by the definition.

To prove (2) it is enough to observe that $C=\bigcup_{\mu \in \mathbb{Z}} \overline{C \cap f^{-1}(\mu)}$.

In (3) it is clear that $\nu(\mathcal{G}(N)) \subset \mathcal{K}(N)$ and that $\nu$ is an additive injection. Suppose that $\nu(\mathcal{G}(N)) \neq \mathcal{K}(N)$ and fix $f \in \mathcal{K}(N) \backslash \nu(\mathcal{G}(N))$. For $g \in \nu(\mathcal{G}(N))$ define

$$
Y(g)=\overline{\{x \in N: f(x) \neq g(x)\}} .
$$

Then $Y(g)$ is a non-empty analytic subset of $N$ and there exists $g_{0}$ such that $\operatorname{dim} Y\left(g_{0}\right)$ is minimal possible. Let $\left\{C_{j}\right\}_{j \in J}$ be the family of all irreducible components of $Y\left(g_{0}\right)$. By (2) there exist "generic values" $\alpha_{j}$ of $f$ and $\beta_{j}$ of $g_{0}$ on each $C_{j}$ for $j \in J$. Define $g_{1}=g_{0}+\sum_{j \in J}\left(\alpha_{j}-\beta_{j}\right) C_{j}$. Then $\operatorname{dim} Y\left(g_{1}\right)<\operatorname{dim} Y\left(g_{0}\right)$, which is impossible.

3. Sequences of positive cycles. Let $N$ be a manifold. In the family $\mathcal{F}_{N}$ of all closed subsets of $N$ we introduce the topology of local uniform convergence generated by the sets

$$
\mathcal{U}(S, K)=\left\{F \in \mathcal{F}_{N}: F \cap K=\emptyset, F \cap U \neq \emptyset \text { for } U \in S\right\}
$$

corresponding to all compact $K \subset N$ and all finite families $S$ of open subsets of $N$. We write $F_{i} \mapsto F$ if $F, F_{i} \in \mathcal{F}_{N}$ for $i \in \mathbb{N}$, and $F$ is the limit of the sequence $\left\{F_{i}\right\}$ in the above topology $\left({ }^{2}\right)$. A detailed study of this convergence can be found in [TW2] (see also [W1] and [Ch], §12.2, §15.5).

Now we wish to investigate the convergence of positive $k$-cycles on $N$ for a fixed $k \in \mathbb{N}$.

(3.1) Definition. We say that a sequence $\left\{Z_{i}\right\}, i \geq 1$, of positive $k$ cycles converges to a positive $k$-cycle $Z_{0}$ (and write $Z_{i} \longmapsto Z_{0}$ ) if:

$(\star)\left|Z_{i}\right| \mapsto\left|Z_{0}\right|$ in the topology of local uniform convergence,

( $\star$ ) for each regular point $a$ of $\left|Z_{0}\right|$ and each submanifold $T$ of $N$ of dimension $n-k$ transversal to $\left|Z_{0}\right|$ at $a$ such that $\bar{T}$ is compact and $\left|Z_{0}\right| \cap \bar{T}=$ $\{a\}$, we have $\operatorname{deg}\left(Z_{i} \cdot T\right)=\operatorname{deg}\left(Z_{0} \cdot T\right)$ for almost all $i\left(^{3}\right)$.

The definition is based on the one given in $[\mathrm{Ch}](\S 12.2, \mathrm{p} .141)$ for the convergence of positive holomorphic chains in open subsets of $\mathbb{C}^{n}$, equivalent

$\left({ }^{2}\right)$ By a standard calculation one can show that $F_{i} \mapsto F$ if and only if for every $x \in F$ and every neighbourhood $V$ of $x, F_{i} \cap V \neq \emptyset$ for sufficiently large $i$, and for every $y \notin F$ there exists a neighbourhood $U$ of $y$ such that $F_{i} \cap U \neq \emptyset$ for at most a finite number of $i$.

$\left({ }^{3}\right)$ Observe that the cycle $Z_{0} \cdot T$ and cycles $Z_{i} \cdot T$ for a sufficiently large $i$ have finite supports and so the degrees (cf. [Dr], [TW3]) are well defined. Recall that for a cycle $A=\sum_{j=1}^{d} \alpha_{j}\left\{a_{j}\right\}, \operatorname{deg}(A)=\sum_{j=1}^{d} \alpha_{j}$. Moreover, $\operatorname{deg}\left(Z_{0} \cdot T\right)=i\left(Z_{0} \cdot T,\{a\}\right)$ is equal to the multiplicity of the component of the cycle $Z_{0}$ containing the point $a$. 
to the convergence in the sense of currents (see [Ch] for details). Notice that in $[R]$ the same convergence is introduced by a metrizable topology.

(3.2) Lemma. Let $k \in \mathbb{N}$ and $Z_{i}$, for $i \in \mathbb{N}$, be positive $k$-cycles. If $\left|Z_{i}\right| \mapsto\left|Z_{0}\right|$, then the following conditions are equivalent:

(1) $Z_{i} \longmapsto Z_{0}$.

(2) For each $a \in\left|Z_{0}\right|$ and each submanifold $T$ of $N$ of dimension $n-k$ such that $\bar{T}$ is compact, $a \in T$ and $\left|Z_{0}\right| \cap \bar{T}=\{a\}$ we have $\operatorname{deg}\left(Z_{i} \cdot T\right)=$ $\operatorname{deg}\left(Z_{0} \cdot T\right)$ for almost all $i$.

(3) For each point a from a given dense subset of regular points of $\left|Z_{0}\right|$ there exists a submanifold $T$ of $N$ of dimension $n-k$ transversal to $\left|Z_{0}\right|$ at a such that $\bar{T}$ is compact, $\left|Z_{0}\right| \cap \bar{T}=\{a\}$ and $\operatorname{deg}\left(Z_{i} \cdot T\right)=\operatorname{deg}\left(Z_{0} \cdot T\right)$ for almost all $i$.

Proof. $(1) \Rightarrow(2)$. Without loss of generality we may assume that $N$ is an open subset of $\mathbb{C}^{n}, a=0$, and $T=\{0\} \times B \subset \mathbb{C}^{k} \times \mathbb{C}^{n-k}=\mathbb{C}^{n}$, where $B$ is the open unit ball in $\mathbb{C}^{n-k}$. There exists an open connected neighbourhood $U$ of $0 \in \mathbb{C}^{k}$ such that $\overline{U \times B} \subset N$ and $(U \times \partial B) \cap\left|Z_{0}\right|=\emptyset$. The mapping $\pi|(U \times B) \cap| Z_{0}|:(U \times B) \cap| Z_{0} \mid \rightarrow U$ is a branched covering and so there exists $x_{0} \in U$ such that $T^{\prime}=\left\{x_{0}\right\} \times B$ and $\left|Z_{0}\right|$ intersect transversally at regular points of $\left|Z_{0}\right|$.

By a standard calculation we have $\operatorname{deg}\left(Z_{0} \cdot T\right)=\operatorname{deg}\left(Z_{0} \cdot T^{\prime}\right)$ and also $\operatorname{deg}\left(Z_{i} \cdot T\right)=\operatorname{deg}\left(Z_{i} \cdot T^{\prime}\right)$ for almost all $i$. Condition (1) implies $\operatorname{deg}\left(Z_{0} \cdot T^{\prime}\right)=$ $\operatorname{deg}\left(Z_{i} \cdot T^{\prime}\right)$ for sufficiently large $i$, and (2) follows.

$(2) \Rightarrow(3)$. Obvious.

$(3) \Rightarrow(1)$. We may assume that:

- $N=B^{\prime} \times \mathbb{C}^{n-k}$, where $B^{\prime}$ is an open unit ball in $\mathbb{C}^{k}$,

- $\left|Z_{0}\right|=B^{\prime} \times\{0\}, a=0$,

- $T=\{0\} \times B$, where $B$ is an open unit ball in $\mathbb{C}^{n-k}$.

There exists $x_{0} \in B^{\prime}$ and a submanifold $T^{\prime}$ which satisfies condition (3) at $\left(x_{0}, 0\right) \in\left|Z_{0}\right|$. Without loss of generality we may assume that $T^{\prime}=F$, where $F: \widetilde{r} B \rightarrow B^{\prime}, \widetilde{r}>0$, is a holomorphic mapping. Fix $r \in(0, \widetilde{r})$ and $r^{\prime} \in(0,1)$ such that $F(r \bar{B}) \subset r^{\prime} B^{\prime}$. Set $T^{\prime \prime}=F \mid(r B)$ and observe that $(3)$ implies $\operatorname{deg}\left(Z_{i} \cdot T^{\prime \prime}\right)=\operatorname{deg}\left(Z_{0} \cdot T^{\prime \prime}\right)=\operatorname{deg}\left(Z_{0} \cdot T\right)=\alpha$ for almost all $i$, where $Z_{0}=\alpha\left|Z_{0}\right|$.

Finally, ([W1], Th. 9.1) gives $\operatorname{deg}\left(Z_{i} \cdot T\right)=\operatorname{deg}\left(Z_{i} \cdot T^{\prime \prime}\right)$ and consequently $\operatorname{deg}\left(Z_{0} \cdot T\right)=\operatorname{deg}\left(Z_{i} \cdot T\right)$ for sufficiently large $i$. This gives (1), and the proof is complete.

Suppose that $F_{i}$, for $i \in \mathbb{N}$, are closed subsets of the manifold $N$. We say that a point $x \in N$ belongs to the upper topological limit of the sequence $\left\{F_{i}\right\}$ (and write $x \in \operatorname{Ls}\left(F_{i}\right)$ ) if and only if each neighbourhood of $x$ intersects 
infinitely many sets from the sequence. Now we are able to formulate the following lemma.

(3.3) Lemma. Suppose that $G$ is a dense subset of a $k$-dimensional connected manifold $D, B$ is an open unit ball in $\mathbb{C}^{n-k}$ and $N=D \times \mathbb{C}^{n-k}$. Let $Z_{i} \neq 0$, for $i \in \mathbb{N}$, be positive $k$-cycles in $N$ such that $\left|Z_{i}\right| \subset D \times B$. If there exists an analytic subset $Z$ of $D \times \mathbb{C}^{n-k}$ of pure dimension $k$ such that $Z \subset D \times B, \operatorname{Ls}\left(\left|Z_{i}\right|\right) \subset Z$ and

$$
\left(\{x\} \times \mathbb{C}^{n-k}\right) \cdot Z_{i} \longmapsto\left(\{x\} \times \mathbb{C}^{n-k}\right) \cdot Z_{0} \quad \text { for } x \in G,
$$

then $Z_{i} \longmapsto Z_{0}$.

Proof. Our assumptions imply that the natural projection $\pi: D \times$ $\mathbb{C}^{n-k} \rightarrow D$ is proper when restricted to each $\left|Z_{i}\right|$. We first prove that $\left|Z_{i}\right| \mapsto$ $\left|Z_{0}\right|$. By ([TW2], Lemma 2) we may assume that $\left\{\left|Z_{i}\right|\right\}$ converges to a closed subset $F=\operatorname{Ls}\left(\left|Z_{i}\right|\right)$ of $N$. It is easy to see that $\left|Z_{0}\right| \subset F \subset Z$. Fix $z_{0} \in F$ and its neighbourhood $V$ in $N$. Since $z_{0}=\left(x_{0}, y_{0}\right) \in Z$, there exist an open connected neighbourhood $U$ of $x_{0}$ in $D$ and a ball $B_{1}$ in $\mathbb{C}^{n-k}$ with centre at $y_{0}$ such that $U \times \bar{B}_{1} \subset V$ and $\left(U \times \partial B_{1}\right) \cap F=\emptyset$. Choose $\widetilde{x} \in U \cap G$ and observe that the assumptions of the lemma imply that $\left(\{\widetilde{x}\} \times \bar{B}_{1}\right) \cap\left|Z_{0}\right| \neq \emptyset$. From what has already been proved, we have $z_{0} \in \overline{\left|Z_{0}\right|}=\left|Z_{0}\right|$. Thus $F=\left|Z_{0}\right|$ and so $\left|Z_{i}\right| \mapsto\left|Z_{0}\right|$.

It remains to prove that condition $(\star \star)$ of Definition (3.1) is satisfied. We have the convergence of supports, and in our situation condition (3) of Lemma (3.2) is satisfied. This completes the proof.

Now, let $N$ be an $n$-dimensional manifold and let $S$ be a closed $s$ dimensional submanifold of $N$. For a given analytic cycle $A=\sum_{j \in J} \alpha_{j} C_{j}$ the part of $A$ supported by $S$ is defined to be

$$
A^{S}=\sum_{j \in J, C_{j} \subset S} \alpha_{j} C_{j} .
$$

Observe that every analytic cycle has a decomposition $A=A^{S}+$ $\left(A-A^{S}\right)$. If $A$ is positive, then both parts of this decomposition are also positive. We will prove the following

(3.4) Theorem. Suppose that $Z_{i}$, for $i \in \mathbb{N}$, are positive $k$-cycles in $N$ such that $Z_{i} \longmapsto Z_{0}$. If $c \in S$ and $\nu\left(Z_{0}^{S}, c\right) \leq \nu\left(Z_{i}^{S}, c\right)$ for $i \in \mathbb{N}$, then there exists an open neighbourhood $U$ of the point $c$ such that:

(1) $Z_{i}^{S} \cap U \longmapsto Z_{0}^{S} \cap U$ and

(2) $\left(Z_{i}-Z_{i}^{S}\right) \cap U \longmapsto\left(Z_{0}-Z_{0}^{S}\right) \cap U$

as sequences of cycles in $U$.

Proof. Without loss of generality we may assume that $k \leq s \leq n$ and that: 
- $N=D \times \mathbb{C}^{s-k} \times \mathbb{C}^{n-s}$, where $D$ is an open connected neighbourhood of $0 \in \mathbb{C}^{k}$,

- $S=D \times \mathbb{C}^{s-k} \times\{0\}$,

- $\left|Z_{i}\right| \subset D \times B$ for $i \in \mathbb{N}$, where $B=B(0,1)$ is the open unit ball in $\mathbb{C}^{n-k}=\mathbb{C}^{s-k} \times \mathbb{C}^{n-s}$,

- $c=0 \in \mathbb{C}^{n}$,

- $\left(\{0\} \times \mathbb{C}^{n-k}\right) \cdot Z_{0}=\nu\left(Z_{0}, 0\right)\{0\}$.

Define $U=N, X_{i}=Z_{i}^{S}, Y_{i}=Z_{i}-Z_{i}^{S}, i \in \mathbb{N}$, and observe that $\operatorname{Ls}\left(\left|X_{i}\right|\right)$ $\subset\left|Z_{0}\right|$ and $\operatorname{Ls}\left(\left|Y_{i}\right|\right) \subset\left|Z_{0}\right|$. Let $G$ be the (dense) subset of $D$ of common regular points of the branched coverings $\pi|| Z_{i}|:| Z_{i} \mid \rightarrow D$, where $i \in \mathbb{N}$ and $\pi: D \times \mathbb{C}^{s-k} \times \mathbb{C}^{n-s} \rightarrow D$ is the natural projection. Let $p: D \times \mathbb{C}^{s-k} \times$ $\mathbb{C}^{n-s} \rightarrow \mathbb{C}^{s-k} \times \mathbb{C}^{n-s}=\mathbb{C}^{n-k}$ be the natural complementary projection.

We will prove the convergence required in the assumptions of the previous lemma for $x \in G$. Fix $x \in G$ and suppose that $p\left(\left|Z_{0}\right| \cap\left(\{x\} \times \mathbb{C}^{n-k}\right)\right)=$ $\left\{y_{1}, \ldots, y_{d}\right\}$. For every $\varepsilon>0$ we have $p\left(\left|Z_{i}\right| \cap\left(\{x\} \times \mathbb{C}^{n-k}\right)\right) \subset \bigcup_{j=1}^{d} B\left(y_{j}, \varepsilon\right)$ for sufficiently large $i$. For $\varepsilon<\varepsilon_{0}$ the closed balls $\bar{B}\left(y_{j}, \varepsilon\right), j=1, \ldots, d$, are pairwise disjoint and we have $\left(\{x\} \times \bar{B}\left(y_{j}, \varepsilon\right)\right) \cap S=\emptyset$ for every $j \notin J=\{l$ : $\left.\left(x, y_{l}\right) \in S\right\}$. The condition $Z_{i} \longmapsto Z_{0}$ implies

$$
\operatorname{deg}\left(\left(\{x\} \times B\left(y_{j}, \varepsilon\right)\right) \cdot Z_{i}\right)=\operatorname{deg}\left(\left(\{x\} \times B\left(y_{j}, \varepsilon\right)\right) \cdot Z_{0}\right)
$$

for $j=1, \ldots, d$ and $i>i_{0}$. If $j \notin J$ we get

$$
\operatorname{deg}\left(\left(\{x\} \times B\left(y_{j}, \varepsilon\right)\right) \cdot Y_{i}\right)=\operatorname{deg}\left(\left(\{x\} \times B\left(y_{j}, \varepsilon\right)\right) \cdot Y_{0}\right)
$$

for $i>i_{0}$, and so $\left(\{x\} \times \mathbb{C}^{n-k}\right) \cdot Y_{i} \longmapsto\left(\{x\} \times \mathbb{C}^{n-k}\right) \cdot Y_{0}$. By Lemma 3.3 we get $Y_{i} \longmapsto Y_{0}$ and (1) follows.

To prove (2) observe that the assumption $\nu\left(X_{0}, 0\right) \leq \nu\left(X_{i}, 0\right)$ for $i \in \mathbb{N}$ implies

$$
\sum_{j \in J} \operatorname{deg}\left(\left(\{x\} \times B\left(y_{j}, \varepsilon\right)\right) \cdot X_{0}\right) \leq \sum_{j \in J} \operatorname{deg}\left(\left(\{x\} \times B\left(y_{j}, \varepsilon\right)\right) \cdot X_{i}\right) .
$$

Moreover, for $i>i_{0}$ we have

$$
\begin{aligned}
\sum_{j \in J} \operatorname{deg}\left(\left(\{x\} \times B\left(y_{j}, \varepsilon\right)\right) \cdot X_{0}\right) & =\sum_{j \in J} \operatorname{deg}\left(\left(\{x\} \times B\left(y_{j}, \varepsilon\right)\right) \cdot Z_{0}\right) \\
& =\sum_{j \in J} \operatorname{deg}\left(\left(\{x\} \times B\left(y_{j}, \varepsilon\right)\right) \cdot Z_{i}\right) .
\end{aligned}
$$

This implies that $\left|Y_{i}\right| \cap\left(\{x\} \times\left(\bigcup_{j \in J} B\left(y_{j}, \varepsilon\right)\right)=\emptyset\right.$ and so, for each $j \in J$ we have

$$
\operatorname{deg}\left(\left(\{x\} \times B\left(y_{j}, \varepsilon\right)\right) \cdot X_{i}\right)=\operatorname{deg}\left(\left(\{x\} \times B\left(y_{j}, \varepsilon\right)\right) \cdot X_{0}\right)
$$

for $i>i_{0}$. This gives $\left(\{x\} \times \mathbb{C}^{n-k}\right) \cdot X_{i} \longmapsto\left(\{x\} \times \mathbb{C}^{n-k}\right) \cdot X_{0}$. By Lemma 3.3 we get $X_{i} \longmapsto X_{0}$ and the proof is complete. 
Now we want to investigate the continuity of intersections of positive analytic cycles. We start with the following lemma:

(3.5) Lemma. Let $S$ be a closed submanifold of a manifold $N$. Suppose that $Z_{i}$, for $i \in \mathbb{N}$, are positive $k$-cycles in $N$ and $Z_{i} \longmapsto Z_{0}$. If $\left|Z_{i}\right|$ intersects $S$ properly for $i \in \mathbb{N}$, then $Z_{i} \cdot S \longmapsto Z_{0} \cdot S$.

Proof. Since $\left|Z_{0}\right|$ intersects $S$ properly, by ([TW2], Th. 3) we get $\left|Z_{i} \cdot S\right| \mapsto\left|Z_{0} \cdot S\right|$. Suppose now that $a$ is a regular point of $V=\left|Z_{0} \cdot S\right|=$ $\left|Z_{0}\right| \cap S$ and $T$ is a submanifold of $S$ of dimension $n-k$ transversal to $V$ at $a$ such that $\bar{T}$ is compact and $\bar{T} \cap V=\{a\}$. Then $\bar{T} \cap\left|Z_{0}\right|=\{a\}$ and by Lemma 3.2 we have $\operatorname{deg}\left(Z_{i} \cdot T\right)=\operatorname{deg}\left(Z_{0} \cdot T\right)$ for sufficiently large $i$. By ([TW3], Th. 2.2) we get $Z_{i} \cdot{ }_{N} T=\left(Z_{i} \cdot{ }_{N} S\right) \cdot{ }_{S} T$ for $i=0,1, \ldots$, and so $\operatorname{deg}\left(\left(Z_{i} \cdot{ }_{N} S\right) \cdot_{S} T\right)=\operatorname{deg}\left(\left(Z_{0} \cdot_{N} S\right) \cdot_{S} T\right)$ for large $i$. This ends the proof.

By the classical "diagonal construction" we finally get

(3.6) TheOREM (cf. [Ch], Corollary 12.3.4). Suppose that $X_{i}$ and $Y_{i}$, for $i \in \mathbb{N}$, are positive $p$ - and $q$-cycles, respectively, in a manifold $M$. If $X_{i} \longmapsto X_{0}, Y_{i} \longmapsto Y_{0}$ and if $\left|X_{i}\right|$ intersects $\left|Y_{i}\right|$ properly for $i \in \mathbb{N}$, then $X_{i} \cdot Y_{i} \longmapsto X_{0} \cdot Y_{0}$.

Proof. Define $Z_{i}:=X_{i} \times Y_{i}$, a $(p+q)$-cycle in $N=M \times M$ for $i \in \mathbb{N}$. Consider the diagonal $S=\Delta_{M}$ of $M \times M$. We have $Z_{i} \longmapsto Z_{0}$ and $\left|Z_{i}\right|$ intersects $S$ properly for $i \in \mathbb{N}$. By the previous lemma we obtain $Z_{i} \cdot S \longmapsto$ $Z_{0} \cdot S$ and this gives the required convergence.

4. Indices of intersection of analytic sets with submanifolds. Let $N$ be an $n$-dimensional manifold. Fix $S$, a closed $s$-dimensional submanifold of $N$, and an open subset $U$ of $N$ such that $U \cap S \neq \emptyset$. Denote by $\mathcal{H}(U)$ the set of all $\mathcal{H}:=\left(H_{1}, \ldots, H_{n-s}\right)$ satisfying the following conditions:

(1) $H_{j}$ is a smooth hypersurface of $U$ containing $U \cap S$ for $j=1, \ldots, n-s$,

(2) $\bigcap_{j=1}^{n-s} T_{x}\left(H_{j}\right)=T_{x} S$ for each $x \in U \cap S$.

For a given analytic subset $Z$ of $N$ of pure dimension $k$ we denote by $\mathcal{H}(U, Z)$ the set of all $\mathcal{H} \in \mathcal{H}(U)$ such that $((U \backslash S) \cap Z) \cap H_{1} \cap \ldots \cap H_{j}$ is an analytic subset of $U \backslash S$ of pure dimension $k-j$ (or empty) for $j=0,1, \ldots, k$.

For every $\mathcal{H}=\left(H_{1}, \ldots, H_{n-s}\right) \in \mathcal{H}(U, Z)$ define an analytic cycle $Z \cdot \mathcal{H}$ in $S \cap U$ by the following

\section{(4.1) Algorithm:}

Step 0: Let $Z_{0}=Z \cap U$. Then $Z_{0}=Z_{0}^{S}+\left(Z_{0}-Z_{0}^{S}\right)$, where $Z_{0}^{S}$ is the part of $Z_{0}$ supported by $S \cap U$.

Step 1: Let $Z_{1}=\left(Z_{0}-Z_{0}^{S}\right) \cdot H_{1}$. Then $Z_{1}=Z_{1}^{S}+\left(Z_{1}-Z_{1}^{S}\right)$, where $Z_{1}^{S}$ is the part of $Z_{1}$ supported by $S \cap U$. 
Step 2: Let $Z_{2}=\left(Z_{1}-Z_{1}^{S}\right) \cdot H_{2}$. Then $Z_{2}=Z_{2}^{S}+\left(Z_{2}-Z_{2}^{S}\right)$, where $Z_{2}^{S}$ is the part of $Z_{2}$ supported by $S \cap U$.

Step $n-s$ : Let $Z_{n-s}=\left(Z_{n-s-1}-Z_{n-s-1}^{S}\right) \cdot H_{n-s}$. Now we have a decomposition $Z_{n-s}=Z_{n-s}^{S}+\left(Z_{n-s}-Z_{n-s}^{S}\right)$, and $\left|Z_{n-s}-Z_{n-s}^{S}\right| \cap S=\emptyset$.

We call a positive analytic cycle $Z \cdot \mathcal{H}=Z_{0}^{S}+Z_{1}^{S}+\ldots+Z_{n-s}^{S}$ in $S \cap U$ the result of the above algorithm.

(4.2) Definition. For $c \in S$ we call

$$
\widetilde{g}(c)=\widetilde{g}(Z, S)(c):=\min _{\operatorname{lex}}\{\widetilde{\nu}(Z \cdot \mathcal{H}, c): \mathcal{H} \in \mathcal{H}(U, Z), c \in U\} \in \mathbb{N}^{s+1}
$$

the extended index of intersection and $g(c)=\widehat{\widetilde{g}(c)}$ the index of intersection of $Z$ with the submanifold $S$ at the point $c$.

5. Index functions. We shall need the following two technical lemmata in the space $\mathbb{C}^{n}$.

(5.1) Lemma. Suppose that $N=\mathbb{C}^{n}$ and $S=\mathbb{C}^{s} \times\{0\}$, where $0 \in$ $\mathbb{C}^{n-s}$. Fix $c \in S, R>0$ and $\mathcal{H}=\left(H_{1}, \ldots, H_{n-s}\right) \in \mathcal{H}(B(c, R))$. Then there exist $r \in(0, R)$, sequences $U_{i}$ of open subsets of $\mathbb{C}^{n}$, and $\mathcal{H}^{(i)}=$ $\left(H_{1}^{(i)}, \ldots, H_{n-s}^{(i)}\right) \in \mathcal{H}\left(U_{i}\right)$ such that $S \cup B(c, r) \subset U_{i}$ for $i=1,2, \ldots$, and $H_{j}^{(i)} \cap B(c, r) \longmapsto H_{j} \cap B(c, r)$ for $j=1, \ldots, n-s$.

Proof. Let $c=\left(x_{0}, 0\right)$ and take $\varrho \in(0, R)$ such that

$$
\begin{aligned}
P_{\varrho} & =\left\{(x, y) \in \mathbb{C}^{s} \times \mathbb{C}^{n-s}:\left|x-x_{0}\right|<\varrho,\left|y_{j}\right|<\varrho \text { for } j=1, \ldots, n-s\right\} \\
& \subset B(c, R)
\end{aligned}
$$

and that there exist functions $h_{j} \in \mathcal{O}\left(P_{\varrho}\right), j=1, \ldots, n-s$, satisfying the conditions $H_{j} \cap P_{\varrho}=h_{j}^{-1}(0), j=1, \ldots, n-s$, and such that $h=$ $\left(h_{1}, \ldots, h_{n-s}\right)$ is a submersion at each point of $S \cap P_{\varrho}$.

One can write

$$
h_{j}(x, y)=\sum_{q=1}^{n-s} y_{q} h_{j, q}(x, y), \quad(x, y) \in P_{\varrho}, j=1, \ldots, n-s,
$$

where $h_{j, q} \in \mathcal{O}\left(P_{\varrho}\right)$. Since $\operatorname{det}\left(\left[h_{j, q}(x, 0)\right]_{j, q=1, \ldots, n-s}\right) \neq 0$ for $x \in B\left(x_{0}, \varrho\right)$, there exist $r \in(0, \varrho / 2)$ and sequences $h_{j, q}^{(i)} \in \mathcal{O}\left(\mathbb{C}^{n}\right)$, for $j, q=1, \ldots, n-s$, such that:

(1) $\operatorname{det}\left[\frac{\partial h}{\partial y}(x, y)\right] \neq 0$ for $(x, y) \in P_{2 r}$,

(2) $\operatorname{det}\left(\left[h_{j, q}^{(i)}(x, 0)\right]_{j, q=1, \ldots, n-s}\right) \neq 0$ for $i=1,2, \ldots$, and $x \in \mathbb{C}^{s}$,

(3) $h_{j, q}^{(i)} \rightarrow h_{j, q}$ uniformly on $P_{2 r}$. 
For $j=1, \ldots, n-s$ define sequences of entire holomorphic functions on $\mathbb{C}^{n}$ by the formulas

$$
h_{j}^{(i)}(x, y)=\sum_{q=1}^{n-s} y_{q} h_{j, q}^{(i)}(x, y),
$$

set $h^{(i)}=\left(h_{1}^{(i)}, \ldots, h_{n-s}^{(i)}\right): \mathbb{C}^{n} \rightarrow \mathbb{C}^{n-s}$, and

$$
U_{i}=\left\{(x, y) \in \mathbb{C}^{n}: \operatorname{det}\left[\frac{\partial h^{(i)}}{\partial y}(x, y)\right] \neq 0\right\} .
$$

Condition (2) above implies $S \subset U_{i}$ for all $i$, and by (1), (3) we get $B(c, r) \subset$ $P_{r} \subset U_{i}$ for sufficiently large $i$. By the definition of $U_{i}, H_{j}^{(i)}:=\left(h_{j}^{(i)}\right)^{-1}(0) \cap$ $U_{i}$ is a smooth hypersurface of $U_{i}$ containing $U_{i} \cap S$ for $j=1, \ldots, n-s$ and $i=1,2, \ldots$ Moreover, $(2)$ gives $\mathcal{H}^{(i)}=\left(H_{1}^{(i)}, \ldots, H_{n-s}^{(i)}\right) \in \mathcal{H}\left(U_{i}\right)$. Since $h_{j}^{(i)} \rightarrow h_{j}$ uniformly on $P_{2 r}$, by Theorem 3.6 we get $H_{j}^{(i)} \cap B(c, r) \longmapsto H_{j} \cap$ $B(c, r)$ for $j=1, \ldots, n-s$. This completes the proof.

(5.2) Lemma. Let $N=B(0,1) \subset \mathbb{C}^{n}, S=B(0,1) \cap\left(\mathbb{C}^{s} \times\{0\}\right)$ and let $Z$ be a pure $k$-dimensional analytic subset of $N$. Fix $c \in S, U=B(c, R) \subset$ $B(0,1)$ where $R>0$, and $\mathcal{H}=\left(H_{1}, \ldots, H_{n-s}\right) \in \mathcal{H}(U, Z)$. Then there exist $r \in(0, R)$, open subsets $U_{i}$ of $N$, and $\mathcal{H}^{(i)}=\left(H_{1}^{(i)}, \ldots, H_{n-s}^{(i)}\right) \in \mathcal{H}\left(U_{i}, Z\right)$ such that $S \cup B(c, r) \subset U_{i}$ for $i=1,2, \ldots$, and $H_{j}^{(i)} \cap B(c, r) \longmapsto H_{j} \cap B(c, r)$ for $j=1, \ldots, n-s$.

P r o of. Take $r \in(0, R)$ and the sequences $\left\{\widetilde{U}_{i}\right\},\left\{\widetilde{\mathcal{H}}^{(i)}\right\}$ constructed by Lemma 5.1 for $\mathcal{H}=\left(H_{1}, \ldots, H_{n-s}\right) \in \mathcal{H}(U, Z) \subset \mathcal{H}(U)$. We may assume that for every $x \in \mathbb{C}^{s}$ the set $\left(\widetilde{U}_{i}\right)_{x}=\left\{y \in \mathbb{C}^{n-s}:(x, y) \in \widetilde{U}_{i}\right\}$ is a ball with centre at 0 in $\mathbb{C}^{n-s}, i=1,2, \ldots$

We can find linear isometries $L_{j}^{(i)}: \mathbb{C}^{n-s} \rightarrow \mathbb{C}^{n-s}$ such that:

(1) $L_{j}^{(i)} \rightarrow \operatorname{id}_{\mathbb{C}^{n-s}}$ as $i \rightarrow \infty$, for $j=1, \ldots, n-s$,

(2) we have $\mathcal{H}^{(i)}=\left(H_{1}^{(i)}, \ldots, H_{n-s}^{(i)}\right) \in \mathcal{H}\left(U_{i}, Z\right)$, where $U_{i}=B(0,1) \cap \widetilde{U}_{i}$ and $H_{j}^{(i)}=\left(\operatorname{id}_{\mathbb{C}^{s}} \times L_{j}^{(i)}\right)\left(\widetilde{H}_{j}^{(i)} \cap B(0,1)\right)$ for $i=1,2, \ldots$ and $j=1, \ldots, n-s$.

It is easy to see that $S \cup B(c, r) \subset U_{i}$ for $i=1,2, \ldots$, and condition (1) implies that

$$
H_{j}^{(i)} \cap B(c, r) \longmapsto H_{j} \cap B(c, r), \quad j=1, \ldots, n-s .
$$

Thus the sequence defined in (2) satisfies the assertions of our lemma and the proof is complete.

In the remainder of this section we fix $N$, an $n$-dimensional manifold, and $S$, a closed $s$-dimensional submanifold of $N$. 
(5.3) Proposition. Let $Z$ be a pure $k$-dimensional analytic subset of $N$. Then for each point $c \in S$ there exists an open neighbourhood $G$ of $c$ in $S$ such that for every $x \in G$ there exist an open subset $U$ of $N$ and $\mathcal{H}=\left(H_{1}, \ldots, H_{n-s}\right) \in \mathcal{H}(U, Z)$ such that $G=U \cap S$ and $\widetilde{g}(x)=\widetilde{\nu}(Z \cdot \mathcal{H}, x)$.

Proof. Fix $c \in S$, an open neighbourhood $V$ of $b$, and a chart $\varphi: V \rightarrow$ $\mathbb{C}^{n}$ such that:

(1) $\varphi(V)=B(0,1), \varphi(c)=0$,

(2) $\varphi(S \cap V)=\left(\mathbb{C}^{s} \times\{0\}\right) \cap B(0,1)$.

We shall prove that under the above conditions we can take $G=S \cap V$. Since $\varphi$ is a biholomorphism, we can restrict our attention to the case $G=$ $S=\left(\mathbb{C}^{s} \times\{0\}\right) \cap B(0,1)$ and $N=B(0,1)$.

Fix $x \in G, R>0$ with $B(x, R) \subset B(0,1)$ and $\widetilde{\mathcal{H}}=\left(\widetilde{H}_{1}, \ldots, \widetilde{H}_{n-s}\right) \in$ $\mathcal{H}(B(x, R), Z)$ such that $\widetilde{g}(x)=\widetilde{\nu}(Z \cdot \widetilde{\mathcal{H}}, x)$. By Lemma 5.2 there exist $r \in(0, R)$, open subsets $U_{i}$ of $N$ and $\mathcal{H}^{(i)}=\left(H_{1}^{(i)}, \ldots, H_{n-s}^{(i)}\right) \in \mathcal{H}\left(U_{i}, Z\right)$ such that $S \cup B(x, r) \subset U_{i}$ for $i=1,2, \ldots$, and $H_{j}^{(i)} \cap B(x, r) \longmapsto H_{j} \cap B(x, r)$ for $j=1, \ldots, n-s$. It is clear that $U_{i} \cap S=G$ for $i=1,2, \ldots$, and so it is enough to prove that for sufficiently large $i$,

$$
\widetilde{\nu}(Z \cdot \widetilde{\mathcal{H}}, x)=\widetilde{\nu}\left(Z \cdot \mathcal{H}^{(i)}, x\right) .
$$

Keeping the notation of Algorithm 4.1 denote by

$$
\begin{aligned}
Z \cdot \widetilde{\mathcal{H}} & =\widetilde{Z}_{0}^{S}+\widetilde{Z}_{1}^{S}+\ldots+\widetilde{Z}_{n-s}^{S}, \\
Z \cdot \mathcal{H}^{(i)} & =\left(Z_{0}^{(i)}\right)^{S}+\left(Z_{1}^{(i)}\right)^{S}+\ldots+\left(Z_{n-s}^{(i)}\right)^{S}, \quad i=1,2, \ldots,
\end{aligned}
$$

its results for $Z$ and $\widetilde{\mathcal{H}}, \mathcal{H}^{(i)}, i=1,2, \ldots$, respectively. Set $\nu_{j}^{(i)}=\nu\left(\left(Z_{j}^{(i)}\right)^{S}, x\right)$ and $\nu_{j}=\nu\left(\widetilde{Z}_{j}^{S}, x\right)$.

After the above preparations, we proceed by induction on $j$. We prove that for each $j=0,1, \ldots, n-s$, there exist $r_{j}>0$ and $i_{j} \in \mathbb{N}$ such that

$$
Z_{j}^{(i)} \cap B\left(x, r_{j}\right) \longmapsto \widetilde{Z}_{j} \cap B\left(x, r_{j}\right) \quad \text { and } \quad \nu_{j}^{(i)}=\nu_{j} \quad \text { for } i \geq i_{j} .
$$

Observe that $Z_{0}^{(i)} \cap B(x, R)=\widetilde{Z}_{0}$ for $i=1,2, \ldots$, and $\nu_{0}^{(i)}=\nu_{0}$ for $i \geq i_{0}=$ 1 , and so $(\star \star)$ is trivially true for $j=0$. Suppose that $j<n-s$ and $(\star \star)$ is true for $0,1, \ldots, j$. We now prove that it also holds for $j+1$.

We know that $Z_{j}^{(i)} \cap B\left(x, r_{j}\right) \longmapsto \widetilde{Z}_{j} \cap B\left(x, r_{j}\right)$ and $\nu_{j}^{(i)}=\nu_{j}$ for sufficiently large $i$. By Theorem 3.4 there exists $r_{j+1} \in\left(0, r_{j}\right)$ such that

$$
\left(Z_{j}^{(i)}-\left(Z_{j}^{(i)}\right)^{S}\right) \cap B\left(x, r_{j+1}\right) \longmapsto\left(\widetilde{Z}_{j}-\widetilde{Z}_{j}^{S}\right) \cap B\left(x, r_{j+1}\right) .
$$

Now Theorem 3.6 implies

$$
Z_{j+1}^{(i)} \cap B\left(x, r_{j+1}\right) \longmapsto \widetilde{Z}_{j+1} \cap B\left(x, r_{j+1}\right) .
$$


Our assumptions imply $\nu_{1}^{(i)}=\nu_{1}, \nu_{2}^{(i)}=\nu_{2}, \ldots, \nu_{j}^{(i)}=\nu_{j}$ for sufficiently large $i$ and

$$
\widetilde{\nu}(Z \cdot \widetilde{\mathcal{H}}, x) \leq_{\text {lex }} \widetilde{\nu}\left(Z \cdot \mathcal{H}^{(i)}, x\right) \quad \text { for all } i \in \mathbb{N} .
$$

Then $\widetilde{\nu}_{j+1} \leq \nu_{j+1}^{(i)}$ for almost all $i$. By Theorem 3.4 there exists an open neighbourhood $U$ of $x$ such that

$$
\left(Z_{j+1}^{(i)}\right)^{S} \cap U \longmapsto\left(\widetilde{Z}_{j+1}\right)^{S} \cap U .
$$

By condition (2) of Lemma 3.2 we get $\nu_{j+1}^{(i)} \leq \widetilde{\nu}_{j+1}$ for almost all $i$.

Summing up, there exists $i_{j+1} \in \mathbb{N}$ such that $\widetilde{\nu}_{j+1}=\nu_{j+1}^{(i)}$ for $i>i_{j+1}$ and $(\star \star)$ is proved.

It is obvious that $(\star \star)$ implies $(\star)$. Thus we can take $\mathcal{H}=\mathcal{H}^{(i)}$ for large enough $i$, and Proposition 5.3 follows.

(5.4) THEOREM. If $Z$ is a pure dimensional analytic subset of $N$, then the extended index function $\widetilde{g}: S \ni x \rightarrow \widetilde{g}(x)=\widetilde{g}(Z, S)(x) \in \mathbb{N}^{s+1}$ is upper semicontinuous in Zariski's topology, when in the image space $\mathbb{N}^{s+1}$ we consider the lexicographic ordering.

Proof. Fix $c \in S$. Let $G$ be a neighbourhood of $c$ from Proposition 5.3 and let $x \in G$. Then

$$
\widetilde{g}(x)=\min _{\operatorname{lex}}\{\widetilde{\nu}(Z \cdot \mathcal{H}, x): \mathcal{H} \in \mathcal{H}(U, Z), G=U \cap S\}
$$

For every $\mathcal{H} \in \mathcal{H}(U, Z)$ such that $G=U \cap S$ the functions $G \ni x \rightarrow$ $\widetilde{\nu}(Z \cdot \mathcal{H}, x) \in \mathbb{N}^{s+1}$ are upper semicontinuous in Zariski's topology. Then $\widetilde{g} \mid G$ is also upper semicontinuous in $G$. It is obvious that semicontinuity can be checked locally (in the standard topology of the submanifold $S$ ), and the theorem follows.

(5.5) THEOREM. If $Z$ is a pure dimensional analytic subset of $N$, then the index function $g: S \ni x \rightarrow g(x)=g(Z, S)(x) \in \mathbb{N} \subset \mathbb{C}$ is analytically constructible.

Proof. By Theorem 5.4 we know that the mapping $\widetilde{g}: S \ni x \rightarrow \widetilde{g}(x) \in$ $\mathbb{N}^{s+1}$ is upper semicontinuous in Zariski's topology of $S$. Then all the fibres of $\widetilde{g}$ are analytically constructible. Observe that for $\alpha \in \mathbb{N}$,

$$
g^{-1}(\alpha)=\bigcup\left\{\widetilde{g}^{-1}(\mu): \widehat{\mu}=\alpha, \mu \in \mathbb{N}^{s+1}\right\},
$$

and so (keeping the notation of Section 2), $g \in \mathcal{K}(S)$. This ends the proof.

6. Intersection theory in complex analytic geometry. Let $X$ and $Y$ be irreducible analytic subsets of a manifold $M$ of dimension $m$ and let $a \in M$. 
(6.1) Definition. The multiplicity of intersection of the sets $X$ and $Y$ at the point $a$ is defined to be

$$
d(a)=d(X, Y)(a)=g\left(X \times Y, \Delta_{M},(a, a)\right) .
$$

Now, we can state the main theorem of this section.

(6.2) TheOREM. The function $M \ni a \rightarrow d(a) \in \mathbb{N} \subset \mathbb{C}$ is analytically constructible.

Proof. By Theorem 5.5 the function

$$
g: \Delta_{M} \ni x \rightarrow g(x)=g\left(X \times Y, \Delta_{M}, x\right) \in \mathbb{C}
$$

is analytically constructible. Since $d(a)=g(a, a)$ it is obvious that $d$ is constructible $(d \in \mathcal{K}(M))$ and the proof is complete.

By Theorem 6.2 and condition (3) of the basic Proposition 2.1 we can state the following definition of the intersection product of the sets $X$ and $Y$.

(6.3) Definition. The intersection product of the irreducible analytic sets $X$ and $Y$ is the unique analytic cycle $X \bullet Y$ in $M$ such that $\nu(X \bullet Y)=$ $d(X, Y)$.

Now, consider two analytic cycles on the manifold $M$ :

$$
X=\sum_{\iota \in I} \alpha_{\iota} X_{\iota}, \quad Y=\sum_{\kappa \in K} \beta_{\kappa} Y_{\kappa} .
$$

It is easy to see that the family of all irreducible components of the cycles $X_{\iota} \bullet Y_{\kappa}, \iota \in I, \kappa \in K$, is locally finite. Then we can state the following

(6.4) Definition. The intersection product of the analytic cycles $X$ and $Y$ is the analytic cycle $X \bullet Y$ in $M$ defined by the (locally finite) sum

$$
X \bullet Y=\sum_{\iota \in I, \kappa \in K} \alpha_{\iota} \beta_{\kappa}\left(X_{\iota} \bullet Y_{\kappa}\right)
$$

In the remainder of this section we assume that $M$ is an $m$-dimensional manifold and $X, Y$ are irreducible analytic subsets of $M$ of dimensions $p$, $q$ respectively. Additionally, define $N=M \times M, S=\Delta_{M}=\{(x, x) \in N$ : $x \in M\}$ and $Z=X \times Y$. We now compare the above definition of the intersection product $X \bullet Y$ with the previous ones in the cases of classical proper intersection and isolated intersection of $X$ and $Y$ in the sense of ([ATW], Definition 5.1).

(6.5) Theorem. If $X$ and $Y$ meet properly on $M$, then $X \bullet Y=X \cdot Y$.

Proof. Fix $a \in M$ and suppose that $X \cdot Y=\sum_{j \in J} \sigma_{j} C_{j}$, where $C_{j}$, $j \in J$, are pairwise distinct irreducible components of $X \cap Y$ and $\sigma_{j}$ denotes the multiplicity of proper intersection along the component $C_{j}$. Since the intersection is proper, $\operatorname{dim} C_{j}=p+q-m$ for $j \in J$. To compute $d(a)$ we have 
to consider the intersection of $Z$ and $S$ at the point $c=(a, a)$. Of course $Z$ and $S$ meet properly and $Z \cdot S=\sum_{j \in J} \sigma_{j} \Delta_{C_{j}}$. Take $\mathcal{H}=\left(H_{1}, \ldots, H_{m}\right) \in$ $\mathcal{H}(U, Z)$, where $U$ is an open neighbourhood of $c$. Then $Z \cdot \mathcal{H}$ is an analytic cycle in $S \cap U$ defined by Algorithm 4.1. Without loss of generality we may assume that $H_{1} \cap \ldots \cap H_{m}=S \cap U$. Then $H_{1} \cdot \ldots \cdot H_{m}=S \cap U$ (with multiplicity 1). By the associativity of proper intersections (cf. [Dr], Th. 5.1, see also [Ch], 12.4, and [TW3], Prop. 2.3) we get $Z_{0}^{S}=\ldots=Z_{m-1}^{S}=\emptyset$ and finally

$$
Z \cdot \mathcal{H}=Z_{m}^{S}=Z \cdot(S \cap U) .
$$

This implies that $\widetilde{\nu}(Z \cdot \mathcal{H}, c)=\left(\mu_{m}, \ldots, \mu_{0}\right)$, where $\mu_{j}=0$ for $j \neq p+q-m$, and $\mu_{p+q-m}=\nu(Z \cdot S, c)$, does not depend on $\mathcal{H} \in \mathcal{H}(U, Z)$ (!). Summing up, $g(c)=g(Z, S)(c)=\nu(Z \cdot S, c)$ and so $d(a)=d(X, Y)(a)=\nu(X \cdot Y, a)$ for all $a \in M$. This implies that $X \bullet Y=X \cdot Y$ and the proof is complete.

(6.6) Theorem. If $a \in M$ and $X \cap Y=\{a\}$, then $X \bullet Y=\sigma\{a\}$, where $\sigma=i(X \cdot Y ; a)$ is the multiplicity of isolated intersection of $X$ and $Y$ at $a$.

Proof. Set $c=(a, a)$ and observe (keeping the notation of [ATW]) that $\sigma=\widetilde{i}(Z \cdot S ; c)$. Take an open neighbourhood $U$ of $c$ and $\mathcal{H}=\left(H_{1}, \ldots, H_{m}\right)$ $\in \mathcal{H}(U, Z)$. Without loss of generality we may assume that the intersection $V=H_{1} \cap \ldots \cap H_{p+q}$ is a submanifold of $N$ (of dimension $2 m-(p+q)$ ) and $V \cap Z=\{c\}$. By the associativity of proper intersections (see the proof of Th. 6.5) we obtain $Z_{j}^{S}=\emptyset$ for $j \neq p+q$ and

$$
Z \cdot \mathcal{H}=Z_{p+q}^{S}=i(Z \cdot V ; c)\{c\} .
$$

Now, ([ATW], Theorem 4.4) implies $\widetilde{g}(c)=\widetilde{g}(Z, S)(c)=\left(\mu_{m}, \ldots, \mu_{0}\right)$, where $\mu_{j}=0$ for $j \neq 0$, and $\mu_{0}=\sigma$. Therefore $d(c)=d(X, Y)(c)=\sigma$. Since $d(a)=0$ for every $x \neq a$, we get $d(x)=\nu(\{a\}, x)$ for $x \in M$. This yields $X \bullet Y=\sigma\{a\}$ and the proof is complete.

\section{References}

[AM1] R. Achilles and M. Manaresi, Multiplicities for improper intersections of analytic subsets, Rend. Sem. Mat. Univ. Pol. Torino 48 (1990), 539-552.

[AM2] - - - Analytic deviation of ideals and intersection theory of analytic spaces, Manuscripta Math. 80 (1993), 291-308.

[AM3] - - - An algebraic characterization of distinguished varieties of intersections, Rev. Roumaine Math. Pures Appl. 38 (1993), 569-578.

[ATW] R. Achilles, P. Tworzewski and T. Winiarski, On improper isolated intersection in complex analytic geometry, Ann. Polon. Math. 51 (1990), 21-36.

[AV] R. Achilles and W. Vogel, On multiplicities for improper intersections, J. Algebra 168 (1994), 123-142.

[Ch] E. M. Chirka, Complex Analytic Sets, Kluwer Acad. Publ., 1989. 
[CT] E. Cygan and P. Tworzewski, Proper intersection multiplicity and regular separation of analytic sets, Ann. Polon. Math. 59 (1994), 293-298.

[De] J. P. Demailly, Courants positifs et théorie de l'intersection, Gaz. Math. 53 (1992), 131-159.

[Dr] R. Draper, Intersection theory in analytic geometry, Math. Ann. 180 (1969), 175-204.

[F] W. Fulton, Intersection Theory, Springer, Berlin, 1984.

[G1] L. J. van Gastel, Excess intersections, Thesis, Univ. of Utrecht, The Netherlands, 1989.

[G2] —, Excess intersections and correspondence principle, Invent. Math. 103 (1991), $197-221$.

[Ł1] S. Łojasiewicz, Ensembles semi-analytiques, I.H.E.S., Bures-sur-Yvette, 1965.

[Ł2] —, Introduction to Complex Analytic Geometry, Birkhäuser, Basel, 1991.

[P1] A. Płoski, Une évaluation pour les sous-ensembles analytiques complexes, Bull. Polish Acad. Sci. Math. 31 (1983), 259-262.

[P2] -, Multiplicity and the Eojasiewicz exponent, preprint 359, Polish Academy of Sciences, Warszawa, 1986

[R] S. Rams, Convergence of holomorphic chains, preprint, 1995.

[S] E. Selder, Eine algebraische Definition lokaler analytischer Schnittmultiplizitäten, Rev. Roumaine Math. Pures Appl. 29 (1984), 417-432.

[SV] J. Stückrad and W. Vogel, An algebraic approach to the intersection theory, in: The Curves Seminar at Queen's University, Vol. II, Queen's Papers in Pure and Appl. Math. 61, Kingston, Ont., 1982, 1-32.

[T] P. Tworzewski, Isolated intersection multiplicity and regular separation of analytic sets, Ann. Polon. Math. 58 (1993), 213-219.

[TW1] P. Tworzewski and T. Winiarski, Analytic sets with proper projections, J. Reine Angew. Math. 337 (1982), 68-76.

[TW2] - - Continuity of intersection of analytic sets, Ann. Polon. Math. 42 (1983), 387-393.

[TW3] - - - Cycles of zeroes of holomorphic mappings, Bull. Polish Acad. Sci. Math. 37 (1989), 95-101.

[V] W. Vogel, Results on Bezout's Theorem, Tata Lecture Notes, Bombay, Vol. 74, Springer, Berlin, 1984.

[Wh] H. Whitney, Complex Analytic Varieties, Addison-Wesley, 1972.

[W1] T. Winiarski, Continuity of total number of intersection, Ann. Polon. Math. 47 (1986), 155-178.

[W2] - Local properties of intersections, in: Deformations of Math. Structures, Kluwer, 1989, 141-150.

INSTITUTE OF MATHEMATICS

JAGIELLONIAN UNIVERSITY

REYMONTA 4

30-059 KRAKÓW, POLAND

E-mail: TWORZEWS@IM.UJ.EDU.PL 